\title{
Construction and protective immunogenicity of DNA vaccine pNMB0315 against Neisseria meningitidis serogroup B
}

\author{
XIAOXIA WU ${ }^{1 *}$, KAIMING LI ${ }^{1,2^{*}}$, MEIHUA XIE $^{1}$, MINJUN YU ${ }^{1}$, \\ SHUANGYANG TANG ${ }^{1}$, ZHENYU LI ${ }^{1}$ and SIHAI HU ${ }^{1}$ \\ ${ }^{1}$ Laboratory of Anti-infectious Immunity, Pathogenic Biology Institute, College of Basic Medicine, University of \\ South China; ${ }^{2}$ Clinical Laboratory, Central Hospital of Hengyang City, Hengyang, Hunan 421001, P.R. China
}

Received February 18, 2017; Accepted October 18, 2017

DOI: $10.3892 / \mathrm{mmr} .2017 .8255$

\begin{abstract}
Neisseria meningitidis ( $N$. meningitidis) is a major cause of meningitis and sepsis. Capsular polysaccharide-based vaccines against serogroups A, C, Y, and W135 are available; however, the development of a vaccine against $N$. meningitidis serogroup B (NMB) has been problematic. NMB0315 is an outer membrane protein of NMB that may be a virulence factor for $N$. meningitidis and a possible target for functional bactericidal antibodies. The present study aimed to develop a potent DNA vaccine against NMB by cloning the NMB0135 gene into the $p c D N A 3.1(+)$ vector to construct the recombinant plasmid pcDNA3.1(+)/NMB0315 (designated pNMB0315). pNMB0315 was transfected into eukaryotic COS-7 and RAW264.7 cells to express the recombinant (r)NMB0315 protein. Protective immunogenicity of the DNA vaccine was assessed in an in vivo mouse model. The levels of rNMB0315-specific immunoglobulin $\mathrm{G}$ ( $\mathrm{IgG}), \operatorname{IgG} 1$ and $\mathrm{IgG} 2 \mathrm{a}$ antibodies in the pNMB0315-immunized group increased dramatically up to week 6 following the initial vaccination, and were significantly higher compared with the levels in the Control groups. The serum concentrations of interleukin- 4 and interferon- $\gamma$ were significantly higher in the $p N M B 0315$-immunized group compared with the control groups. Following intraperitoneal challenge with a lethal dose of NMB strain MC58, the survival rate in the $p N M B 0315+\mathrm{CpG}$ group was $70 \%$ (14 out of 20 mice) at 14 days; by contrast, all mice in the control groups succumbed within 3 days. The serum bactericidal titers of the $p N M B 0315+\mathrm{CpG}$ group in vitro reached 1:128 following
\end{abstract}

Correspondence to: Professor Sihai Hu, Laboratory of Anti-infectious Immunity, Pathogenic Biology Institute, College of Basic Medicine, University of South China, 28 West Changsheng Road, Hengyang, Hunan 421001, P.R. China

E-mail: hhsshh_518@163.com

${ }^{*}$ Contributed equally

Key words: Neisseria meningitidis serogroup B, outer membrane protein 0315 , DNA vaccine, immunogenicity, immunoprotection three immunizations. The results indicated that $p N M B 0315$ may serve as a promising DNA vaccine against NMB.

\section{Introduction}

Neisseria meningitidis ( $N$. meningitidis) is a Gram-negative microorganism and a major causative agent of severe sepsis and meningitis (1), both of which may lead to mortality in children and young adults within h, although effective antibiotics are available $(2,3)$. $N$. meningitidis causes meningococcal diseases in $~ 500,000$ people annually worldwide, mainly affecting children between the ages of 3 and 48 months, followed by adolescents (4), and although drug treatments such as penicillin are available, $10-15 \%$ of children and adolescents succumb to infection. Of those who survive, 11-19\% exhibit problems in the nervous system, including mental retardation, hearing loss and paralysis (5). N. meningitidis is also responsible for the development of invasive meningococcal diseases, such as septicemia, pneumonia and arthritis (6).

N. meningitidis is classified into 13 serogroups $(\mathrm{A}, \mathrm{B}, \mathrm{C}$, D, H, I, K, L, X, Y, Z, 29E and W-135) based on the molecular structure and antigenicity of bacterial capsular polysaccharides (CPSs) (3); among the serogroups A-C cause $\leq 90 \%$ of all meningitis cases. CPS-based vaccines have been developed and used successfully to prevent the invasive meningococcal disease that may be caused by serogroups A, C, W135 and $\mathrm{Y}$ (7). However, the CPS of $N$. meningitidis serogroup B (NMB) is an $\alpha_{2-8}$-linked polysialic acid that resembles a molecule that is present on the surface of human tissues, thus making a NMB CPS-based vaccine poorly immunogenic, as well as presenting a possible cause of autoimmunity $(2,7,8)$.

Over the past 40 years there has been an increase in the number of studies directed towards the identification of NMB antigens as a basis of developing a new vaccine. Outer membrane proteins of $N$. meningitidis have been implicated in bacterial virulence and may induce immune responses; therefore, they may present good antigen candidates for vaccine design (9).

NMB outer membrane protein 0315 (NMB0315; NCBI Gene ID: 902431) was confirmed as an outer membrane protein of NMB that comprises 430 amino acids and has a molecular weight of $46 \mathrm{kDa}$, and has been demonstrated to be a virulence factor for $N$. meningitidis and a target for 
bactericidal antibodies (10). A previous study revealed that certain proteins that were $\leq 98 \%$ identical to NMB0315 were not only in the different serogroups of $N$. meningitidis, but also in Neisseria gonorrhoeae (10), which suggested that NMB0315 may be a potential candidate as a broad-spectrum vaccine against meningococcal diseases.

An NMB0315 DNA vaccine was constructed. The NMB0315 gene was amplified by polymerase chain reaction (PCR) from the NMB MC58 standard strain genomic DNA (NCBI accession no. NC_003112.2) and cloned into a pcDNA3.1(+) plasmid to construct a recombinant plasmid, pcDNA3.1(+)/NMB0315 (designated pNMB0315). The constructed $p N M B 0315$ was transfected into eukaryotic COS-7 and RAW264.7 cells to express the recombinant NMB0315 (rNMB0315) protein. To determine the immunogenicity and protective efficacy of $p N M B 0315$, female BALB/c mouse were used as an in vivo model. The levels of NMB0315-specific immunoglobulin $\mathrm{G}$ (IgG), IgG1 and IgG2a antibodies that were induced by $p c D N A 3.1(+) / N M B 0315$ were detected, and the protective immunogenicity was evaluated to develop a novel DNA vaccine against NMB.

\section{Materials and methods}

Animals and housing. Pathogen free, female BALB/c inbred mice (125 mice, 3-4 weeks, average weight, $19.8 \mathrm{~g}$ ) were purchased from The National Resource Center for Rodent Laboratory Animal (Shanghai, China). Animals were maintained in the animal facilities of The University of South China (Hengyang, China), and raised on a normal diet (food and water were available ad libitum) at $25^{\circ} \mathrm{C}$ and $50 \%$ humidity on a 12-h light/dark cycle prior to euthanasia. All experimental protocols involving mice were approved by the Ethics Committee of The University of South China.

Plasmids, bacterial strains, cell lines and reagents. The pcDNA3.1(+) plasmid (Addgene, Inc., Cambridge, MA, USA) and Escherichia coli strain JM109 (China Center of Industrial Culture Collection, $\mathrm{CICC}^{\circledR}$; Beijing, China) were used in conventional recombinant experiments. NMB strain MC58 was purchased from American Type Culture Collection (ATCC; Manassas, VA, USA). Neisseria mucosa was separated from a normal population. COS-7 monkey kidney fibroblast (commonly used for the production of recombinant proteins) and RAW264.7 mouse leukemia cell lines (commonly used model of mouse macrophages for the study of cellular responses to microbes and their products) were purchased from Institute of Cell Biology of the Chinese Academy Sciences (Shanghai, China). Restriction enzymes $B a m$ HI and XhoI, T4 DNA ligase, Pfu DNA polymerase, pre-stained protein molecular weight marker, nuclease-free water and dNTP Mix were purchased from Thermo Fisher Scientific, Inc. (Waltham, MA, USA). CpG (sequence TCC ATGACGTTCCTGACGTT) was synthesized by Beijing Genomics Institute (Shenzhen, China). Horseradish peroxidase (HRP)-conjugated goat anti-mouse IgG (cat. no. ab97023), IgG1 (cat. no. ab97240), IgG2a (cat. no. ab97245) antibodies were all purchased from Abcam (Cambridge, UK). ELISA kits (cat. nos. IFN-r/88-7314-22 and IL-4/88-7044-22) were purchased from eBioscience (Thermo Fisher Scientific, Inc.).
N. meningitidis Serogroup B Diagnostic Antiserum from BD Biosciences (Franklin Lakes, NJ, USA) (3).

Construction and identification of pcDNA3.1(+)/NMB0315. $B a m \mathrm{HI}$ and $\mathrm{XhoI}$ were selected as the restriction sites for the upstream and downstream primers, respectively. The full length NMB0315 gene (GI: 902431, location 326,142-327,434, length $1293 \mathrm{bp}$ ) was amplified by PCR (pre-denaturation at $94^{\circ} \mathrm{C}$, for $2 \mathrm{~min}$, denaturation at $94^{\circ} \mathrm{C}$, for $30 \mathrm{sec}$; annealing at $60^{\circ} \mathrm{C}$ for, $30 \mathrm{sec}$ and extension at $72^{\circ} \mathrm{C}$, for $2 \mathrm{~min}, 34$ cycles; a final extension $/ 72^{\circ} \mathrm{C}$ for $10 \mathrm{~min}$. DNA polymerase, nuclease-free water and dNTP Mix were purchased from Thermo Fisher Scientific, Inc. Successful amplification was confirmed using $1 \%$ agarose gel, stained with Gold View (cat. no. 200601; BLKW Biotechnology Co., Ltd., Beijing, China) using the NMB strain MC58 genomic DNA (extracted by Bacteria Genomic DNA kit; CoWin Bioscience Co., Ltd., Beijing, China; cell density $1 \times 10^{6}$ cells $/ \mathrm{ml}$ ) as a template and the upstream primer (BamHI), 5'-CGCGGATCCATGGCT GTCTTCCCACTTTC-3' and downstream primer (XhoI), 5'-CCGCTCGAGTCAATCCGATTGCGACAC-3' were used. The amplified PCR product was digested with restriction enzymes BamHI and XhoI (1 $\mu \mathrm{g}$ PCR product, $0.4 \mu \mathrm{M} \mathrm{BamHI}$, $0.4 \mu \mathrm{M} \mathrm{Xhol} / 25 \mu \mathrm{l})$ and cloned into $p c D N A 3.1(+)$ to generate a recombinant plasmid $p c D N A 3.1(+) / N M B 0315$ ( $p N M B 0315)$, which was used as a DNA vaccine. The constructed recombinant $p N M B 0315$ was confirmed by restriction digestion and sequencing, and subsequently transformed (200 $\mu$ l competent $\mathrm{JM} 109 / \mathrm{OD}_{600}=0.6$ plus $10 \mathrm{ng} p c D N A 3.1(+) / N M B 0315$ plasmid were transformed at room temp for $30 \mathrm{~min}$ then $42^{\circ} \mathrm{C}$ for $90 \mathrm{sec}, 4^{\circ} \mathrm{C}$ for $3 \mathrm{~min}$. Then $800 \mu \mathrm{l} \mathrm{LB}$ broth was added and incubated at $37^{\circ} \mathrm{C}$ for $1 \mathrm{~h}$ followed by centrifugation at $4^{\circ} \mathrm{C}$, $4,000 \mathrm{x}$ g for $60 \mathrm{sec}$. The positive transformants were selected on LB agar containing ampicillin into E. coli JM109 for overexpression. The clones containing the insert $N M B 0315$ were selected by resistance to ampicillin and stored for further use.

Expression and identification of rNMB0315. Eukaryotic COS-7 and RAW264.7 cell lines were cultured in Dulbecco's modified Eagle's medium (Thermo Fisher Scientific, Inc.) supplemented with $10 \%$ heat-inactivated fetal bovine serum (Gibco; Thermo Fisher Scientific, Inc.) at $37^{\circ} \mathrm{C}$ in a $5 \% \mathrm{CO}_{2}$. The cell lines $\left(2 \times 10^{5} / \mathrm{ml}\right)$ were transfected with $p N M B 0315$ $(8 \mu \mathrm{g} / \mathrm{ml})$ in a 24 -well plate at room temperature for $48 \mathrm{~h}$ using the X-treme GENE HP DNA Transfection Reagent (Roche Diagnostics, Shanghai, China), according to the manufacturer's instructions. COS-7 or RAW264.7 cells were transfected with empty vector $p c D N A 3.1(+)$ or PBS as controls. The transfected cells were harvested $48 \mathrm{~h}$ post-transfection to evaluate the expression of rNMB0315 by immunocytochemical method and western blot analysis. For immunocytochemical method and western blot analysis, rabbit immune serum-containing antibodies of recombinant rNMB0315 (prepared by the authors' group, prokaryotic expression product) were used as primary antibody $(1: 1,000)$ and HRP-conjugated goat anti-rabbit IgG as secondary antibody (1:5,000, cat. no. SC-2357; Santa Cruz Biotechnology, Inc., Dallas, TX, USA).

Immunocytochemistry. COS-7 or RAW264.7 cells $\left(10^{6} / \mathrm{ml}\right)$ were cultured in Dulbecco's modified Eagle's medium 
containing $10 \%$ fetal bovine serum (Gibco; Thermo Fisher Scientific, Inc.), at $37^{\circ} \mathrm{C}$ in a humidified atmosphere containing $5 \% \mathrm{CO}_{2}$. Cells were washed three times with pre-cooled PBS and seeded onto glass coverslips (Thermo Fisher Scientific, Inc). The cells were fixed with $4 \%$ paraformaldehyde (Sigma-Aldrich; Merck KGaA, Darmstadt, Germany) for $20 \mathrm{~min}$ at room temperature and then washed three times with PBS prior to the addition of $3 \% \mathrm{H}_{2} \mathrm{O}_{2}$ for 10 min (inhibitor of endogenous peroxidase activity). Blocking was performed with 5\% bovine serum albumin (BSA; Gibco; Thermo Fisher Scientific, Inc.) for $30 \mathrm{~min}$ at $37^{\circ} \mathrm{C}$, incubated the primary antibody [1:1,000; preparation of primary antibody: New Zealand rabbits (6 rabbits, 6 months, female, $2-2.5 \mathrm{~kg}$, maintained under specific pathogen-free conditions in isolated cages, with a $12-\mathrm{h}$ light-dark cycle at $25^{\circ} \mathrm{C}, 50 \%$ humidity and ventilation facility, food and water were available ad libitum)] were immunized with rNMB0315 protein $(200 \mu \mathrm{g} / 200 \mu \mathrm{l}$ each time, prepared by the authors' group) in subcutaneous injection above the gluteals, with Freund's adjuvant (Sigma-Aldrich; Merck KGaA) at 0 week (complete Freund's adjuvant; cat. no. F5881), 2, 4 and 6 weeks (incomplete Freund's adjuvant; cat. no. F5506); two weeks following the last immunization, blood was collected via ear vein and centrifuged $(800 \mathrm{x} \mathrm{g})$ at room temperature for $20 \mathrm{~min}$, the serum was stored at $-70^{\circ} \mathrm{C}$ ] at $4^{\circ} \mathrm{C}$ overnight, washed three times with PBS. HRP-conjugated goat anti-rabbit $\operatorname{IgG}$ as secondary antibody (1:5,000; Abcam, Cambridge, UK) was added for $60 \mathrm{~min}$ at room temperature, then washed three times. Stained with DAB for $1 \mathrm{~min}$, at room temperature and then with hematoxylin (both from Sigma-Aldrich; Merck $\mathrm{KGaA}$ ) for $8 \mathrm{~min}$, at room temperature was performed. Images were captured using an inverted microscope (XD202; Nanjing Jiangnan Novel Optical Co., Ltd., Nanjing, China). A total of 50 fields were examined (magnification, x200).

Western blotting. COS-7 or RAW264.7 cells $\left(10^{6} / \mathrm{ml}\right)$ were harvested $48 \mathrm{~h}$ following transfection and resuspended in lysis buffer [50 mM Tris (pH 7.5), $150 \mathrm{mM} \mathrm{NaCl}, 5 \mathrm{mM}$ EDTA and 1\% NP-40] supplemented with Complete Mini (Roche Diagnostics) for protein extraction. Protein concentrations were determined using the bicinchoninic acid Protein Assay kit (Beyotime Institute of Biotechnology, Shanghai, China). Protein extracts $(10 \mu \mathrm{g} / 10 \mu \mathrm{l} /$ lane) were separated by $10 \%$ SDS-PAGE, transferred to polyvinylidene fluoride membrane (Beyotime Institute of Biotechnology) blocked by $1 \%$ BSA for $3 \mathrm{~h}$ at room temperature. The membranes were incubated with the primary antibody $(1: 1,000)$ anti-NMB0315 (prepared by the authors' group) at $4^{\circ} \mathrm{C}$ overnight then washed three times with PBS-T. $\beta$-actin was used for internal control $(\beta$-actin rabbit polyclonal antibody, cat. no. 20536-1-AP, 1:3,000; Proteintech, Group, Inc., Chicago, IL, USA). HRP-conjugated goat anti-rabbit IgG was added as a secondary antibody $(1: 5,000$; Abcam) for $45 \mathrm{~min}$ at room temperature, washed three times. Signals detection was performed with an enhanced chemiluminescence kit (GE Healthcare, Chicago, IL, USA).

Animal immunization and specimen collection. A total of 125 4-week-old female BALB/c mice were randomly and equally divided into five groups ( 25 mice/group), and were subsequently immunized intramuscularly as follows (total volume of $100 \mu \mathrm{l}$ each group): i) $p N M B 0315(50 \mu \mathrm{g})+\mathrm{CpG}(10 \mu \mathrm{g}$; as an adjuvant); ii) $p N M B 0315$-alone $(50 \mu \mathrm{g})$; iii) $p c D N A 3.1(+)$ (50 $\mu \mathrm{g})$; iv) CpG (10 $\mu \mathrm{g})$; or v) PBS. The inoculation was performed three times with an interval of 2 weeks four times (0, 2, 4 and 6 weeks), and blood samples were collected from the immunized mice by tail bleeding prior to each immunization. The collected serum was stored at $-70^{\circ} \mathrm{C}$ until use.

Specific antibody assay. NMB0315-specific IgG, IgG1 and IgG2a antibody levels were determined by indirect ELISA as previously described (11). Briefly, ELISA plates were coated with $1 \mu \mathrm{g} /$ well of purified rNMB0315 (prokaryotic expression product), sealed and incubated overnight at $37^{\circ} \mathrm{C}$. Following 3 washes with PBS $+0.05 \%$ Tween-20 (PBST), the coated plates were blocked with $150 \mu$ l blocking buffer $(0.5 \%$ skim milk in PBST) at $37^{\circ} \mathrm{C}$ for $1 \mathrm{~h}$. The plate was washed 3 times with PBST and incubated with $100 \mu 1$ NMB0315-immune mouse serum (1:10,000 in blocking buffer was used as a working dilution following testing of serial dilutions) each well in a 96 well plate for $1 \mathrm{~h}$ at room temperature. A blank control without serum was set up concurrently. Following 3 washes, $100 \mu 1$ HRP-conjugated goat anti-mouse IgG, IgG1 or IgG2a secondary antibody (1:5,000 in PBST) was added to the respective ELISA plate wells, and the plates were incubated at room temperature in the dark for $30 \mathrm{~min}$. The plates were washed 5 times, $100 \mu 1$ 3,3,5,5-tetramethylbenzidine Microwell Peroxidase Substrate System (Tiangen Biotech Co., Ltd., Beijing, China) was added and the plates were incubated $37^{\circ} \mathrm{C}$ for $20 \mathrm{~min}$. The reactions were stopped with the addition of $100 \mu 1 \mathrm{H}_{2} \mathrm{SO}_{4}(2 \mathrm{~mol} / \mathrm{l})$ to each well, and signal detected using a Bio-Rad microplate reader (Bio-Rad Laboratories, Inc., Hercules, CA, USA) at an absorbance of $450 \mathrm{~nm}$. All assays were performed in triplicate. The cutoff value was set as previously described (12).

Cytokine assays. A total of 5 immunized mice from each group were euthanized 2 weeks following the third immunization, and their spleens were isolated for preparation of splenic suspension. The spleen from the immunized mice were obtained using aseptic techniques, ground and filtered by 200-mesh sieve (70- $\mu \mathrm{M}$ pore size) and prepared for single-cell suspension. The suspension was used to seed 96-well plates $\left(1 \times 10^{6}\right.$ cells/well), and the cells were incubated with rNMB0315 $(10 \mu \mathrm{g})$, as a specific stimulator, at $37^{\circ} \mathrm{C}$ in $5 \% \mathrm{CO}_{2}$ for $48 \mathrm{~h}$. The culture supernatant was collected and the levels of $\mathrm{T}$ helper 1 (Th1)- and Th2-type cytokines, interleukin (IL)-4 and interferon (IFN)- $\gamma$, respectively, were detected by indirect ELISA, method according to the manufacturer's instructions.

Serum bactericidal assay (SBA). Two weeks following the third immunization (20 mice in each group), immune serum was collected for SBA as described previously (3). Briefly, a suspension of MNB strain MC58 (40,000 CFU/ml) was mixed with newborn rabbit complement (Pel-Freez Biologicals, Rogers, AR, USA) at a 1:1 ratio; the mixture was subsequently combined with the immune serum, serially diluted in 2-fold from 1:2 to $1: 256$, and cultured for $1 \mathrm{~h}$ at $37^{\circ} \mathrm{C}$ prior to being inoculated on chocolate agar plates and incubated at $37^{\circ} \mathrm{C}$ overnight. MNB diagnostic antiserum was used as a positive control; negative controls included MC58 suspension + complement, UV-inactivated MC58 + complement, and 
A

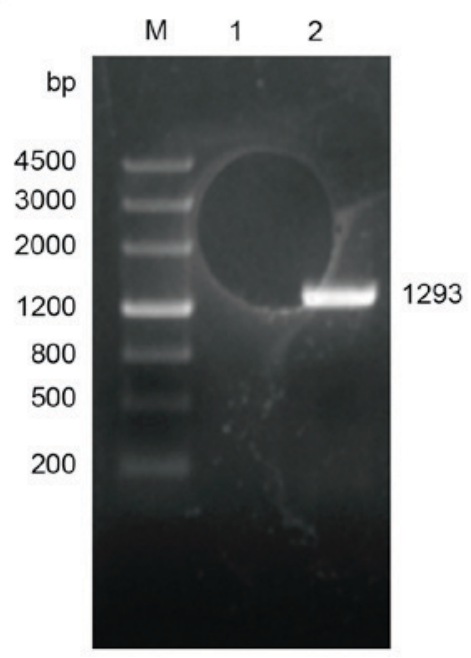

$B$

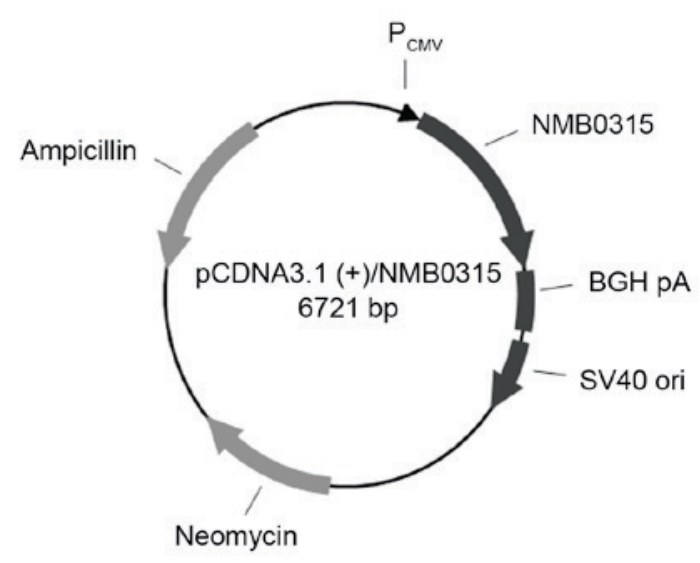

Figure 1. Construction of $p N M B 0315$. (A) PCR amplification of NMB0315 gene from MC58. M, DNA marker; lane 1, control (Neiserria mucosa); lane 2, NMB0315 PCR product (1,293 bp). (B) Diagram representation of the recombinant plasmid $p N M B 0315 . \mathrm{P}_{\mathrm{CMV}}$ is a human CMV immediate-early promoter for high-level expression in eukaryotic cells. NMBO315 is the target gene of the protective antigen against NMB. BGH pA is an mRNA 3'-untranslated region element for supporting transgene mRNA stability and regulating in mRNA translation. SV40 ori is for episomal replication and simple vector rescue in cell lines. The neomycin resistance gene is responsible for the selection of stable cell lines and the ampicillin resistance gene is for selection and maintenance in Escherichia coli. BGH pA, bovine growth hormone polyadenylation sequence; CMV, cytomegalovirus; NMB, Neisseria meningitidis serogroup B; SV40 ori, simian virus 40 origin site.

immune serum + MC58 + heat-inactivated complement. When the serum with a bactericidal rate (determined by counting of the cultured bacteria colonies, compared with the negative and positive controls) was $>50 \%$, the highest serum dilution was determined using serum bactericidal antibody titers (3).

Immunoprotection of pNMB0315. Cultured MC58 cells were diluted to an optical density with $\mathrm{A}_{600}=0.005$ (a concentration equivalent to 4,000 colony-forming units/ml, which is a lethal dose of MC58; mice were infected with 500, 1,000, 2,000, 3,000, 4,000 colony-forming units/ml MC58 respectively, a lethal dose resulted in mice succumbing in $72 \mathrm{~h}$ was confirmed) to form the strain suspension. The MC58 suspension $(40,000 \mathrm{CFU} / \mathrm{ml})$ was immediately injected into the abdominal cavity of immunized mice (20 mice in each group), 2 weeks following the third immunization. Signs of MC58 infection and survival rate of the mice were recorded daily for 14 days, following which all mice were humanely euthanized with $100 \% \mathrm{CO}_{2}$.

Statistical analysis. Data are expressed as the mean \pm standard deviation. SPSS 18.0 statistical software (SPSS, Inc., Chicago, IL, USA) was used for one-way analysis of variance for data sets containing multiple comparisons and a Dunnett's test as a post-hoc test. Kaplan-Meier with log-rank test was used for comparison of the survival rate of mice. $\mathrm{P}<0.05$ was considered to indicate a statistically significant difference.

\section{Results}

Construction of a recombinant plasmid pNMB0315. The full-length NMB0315 gene sequence from the genomic DNA of NMB standard strain MC58 was amplified by PCR, and a NMB0315 gene (1,293 bp) was obtained (Fig. 1A). The NMB0315 gene was cloned into the mammalian expression vector $p c D N A 3.1(+)$ to generate the recombinant plasmid pNMB0315 (Fig. 1B).

Identification of pNMB0315. The constructed recombinant plasmid pNMB0315 (total length, 6,721 bp) was identified by double digestion of the recombinant plasmid with restriction enzymes BamHI and XhoI, followed by sequencing (data not shown). The digested products were $1,293 \mathrm{bp}$, which corresponded to the NMB0315 gene insert, and 5,428 bp, which corresponded to the $p c D N A 3.1(+)$ plasmid vector (Fig. 2A). The recombinant plasmid $p N M B 0315$ was transfected into eukaryotic COS-7 and RAW264.7 cells, and the expressed NMB0315 protein was identified by immunocytochemical method and western blot analysis (Fig. 2B and C, respectively). Detection was similar for RAW264.7 cells (data not shown). For immunocytochemical method and for western blotting, rabbit immune serum containing NMB0315-specific antibodies was used as the primary antibody and HRP-conjugated goat anti-rabbit IgG as the secondary antibody. Cells transfected with $p N M B 0315$ were stained brown, indicating the detection of rNMB0315 expression (Fig. 2B-a), whereas no rNMB0315 protein was detected in the control cells (Fig. 2B-b-d). The recombinant plasmid $p N M B 0315$ was transfected into eukaryotic COS-7 and RAW264.7 cells and the expressed NMB0315 protein was identified by western blot analysis (Fig. 2C). The results indicated that a eukaryotic recombinant plasmid pNMB0315 was successfully constructed and could effectively expressed rNMB0315 protein in mammalian cells.

Humoral immune response is induced by pNMB0315. The levels of NMB0315-specific IgG, IgG1 and IgG2a antibodies in the $p N M B 0315+\mathrm{CpG}$ group and the $p N M B 0315$-only group notably increased between week 2 and week 6 following the initial vaccination, and were significantly higher compared with the respective antibody levels in the control groups, 
A

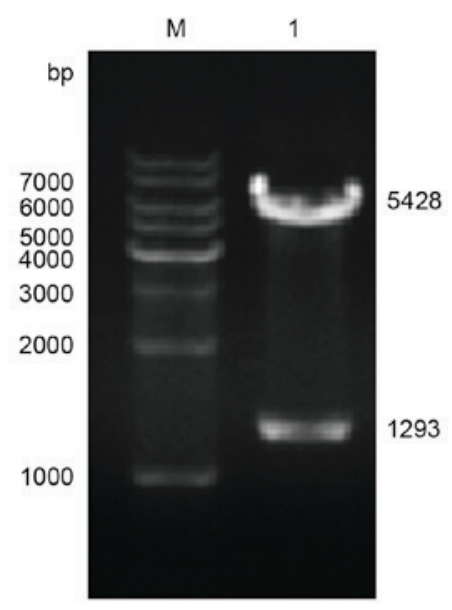

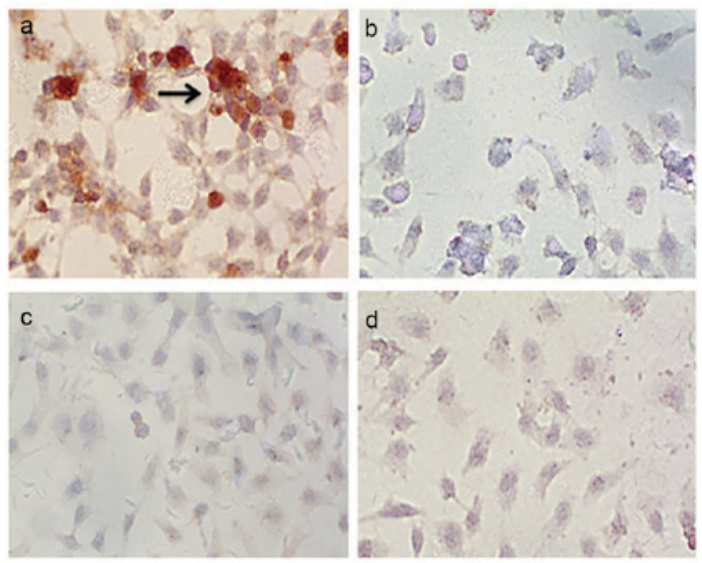

C

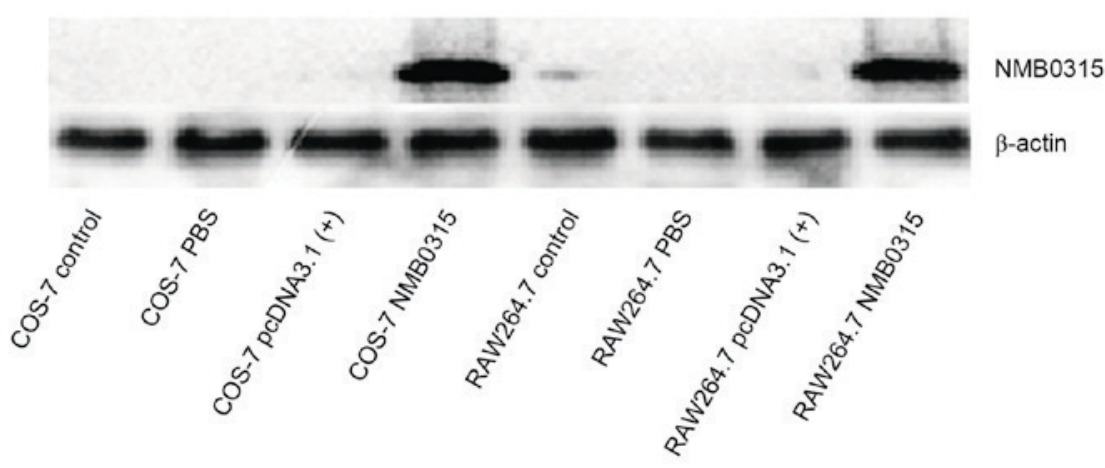

Figure 2. Enzyme digestion, immunocytochemical staining and western blot analysis of $p N M B 0315$. (A) Recombinant plasmid $p N M B 0315$ was digested with $B a m H I$ and $X h o I$ to confirm successful cloning of the $N M B 0315$ gene into the plasmid vector. M, DNA marker; lane 1, digested fragments of $p N M B 0315$, in which the 5,428 bp fragment is the pcDNA3.1(+) vector, and the 1,293 bp fragment is NMB0315. (B) Expression of NMB0315 protein in eukaryotic COS-7 cells by immunocytochemical assay; magnification, x200. (B-a) COS-7 cells transfected with $p N M B 0315$; (b) COS-7 cells transfected with $p c D N A 3.1(+)$; (c) COS-7 cells transfected with PBS; and (d) untransfected COS-7 cells. (C) Eukaryotic expression of recombinant NMB0315 protein was detected by western blot analysis. COS-7 control, untransfected COS-7 cells; COS-7 PBS, cells transfected with PBS; COS-7 pcDNA3.1(+), cells transfected with pcDNA3.1(+); COS-7 NMB0315, cells transfected with pNMB0315; RAW264.7 control, untransfected RAW264.7 cells; RAW264.7 PBS, cells transfected with PBS; RAW264.7 pcDNA3.1(+), cells transfected with $p c D N A 3.1(+)$; RAW264.7 NMB0315, cells transfected with pNMB0315. NMB, Neisseria meningitidis serogroup B .

pcDNA3.1(+), PBS and CpG-only at weeks 2, 4 and $6(\mathrm{P}<0.01$; Fig. 3). The specific IgG, IgG1 and IgG2a antibody levels in the $p N M B 0315+\mathrm{CpG}$ group were significantly higher compared with the levels in the $p N M B 0315$-only group at weeks 4 and $6(\mathrm{P}<0.05$; Fig. 3$)$. It has been reported previously that IgG2a predominantly indicates cellular immunity and $\mathrm{IgG} 1$ predominantly indicates humoral immunity (3); therefore, the serum IgG2a/IgG1 ratios were calculated in the pNMB0315-only group at weeks 2,4 and 6 post-immunization were $0.775(0.152 / 0.196), 0.744(0.253 / 0.340)$ and 0.614 (0.309/0.503), respectively; in the $p N M B 0315+\mathrm{CpG}$ group at weeks 2,4 and 6 post-immunization 0.595 (0.084/0.141), 0.723 $(0.186 / 0.257)$ and $0.565(0.234 / 0.414)$, respectively. All ratios were $<1$, which suggested that the DNA vaccine $p N M B 0315$ predominantly induced humoral immunity responses.

Th1-type and Th2-type cytokines are induced by pNMB0315. Splenic cells from the immunized mice were harvested 2 weeks following the third immunization, and were subsequently stimulated in vitro with $10 \mu \mathrm{g} / \mathrm{ml}$ of rNMB0315 protein for $48 \mathrm{~h}$. The levels of IL-4 and IFN- $\gamma$ in the splenic lymphocytes culture supernatant were detected by indirect ELISA. The concentrations of IL-4 and IFN- $\gamma$ in the $p N M B 0315+\mathrm{CpG}$ group and in the $p N M B 0315$-only group were significantly higher compared with the respective expression levels in the $p c D N A 3.1(+)$, PBS and $\mathrm{CpG}$ control groups $(\mathrm{P}<0.01 ; \mathrm{Fig}$. $4 \mathrm{~A}$ and $\mathrm{B})$. In addition, the concentrations of IL- 4 and IFN- $\gamma$ in the $p N M B 0315+\mathrm{CpG}$ group were significantly higher compared with the $p N M B 0315$-only group $(\mathrm{P}<0.05$; Fig. 4). These results suggested that $p N M B 0315$ may elicit a potent Th1-type cytokine (IFN- $\gamma$ ) and Th2-type cytokine (IL-4) response in the immunized mice.

Immunoprotection efficacy against challenge with $N M B$ $M C 58$. Two weeks following the third vaccination, immunized mice were challenged with a lethal dose of NMB MC58. All mice in the pcDNA3.1(+), PBS and CpG control groups displayed signs of severe clinical symptoms, including rough hair, shivering, decreased mobility and severe ataxia, and succumbed to the infection within 3 days (Fig. 5). Survival rates in the $p N M B 0315$-only group and in the $p N M B 0315+\mathrm{CpG}$ group were 65\% (13 out of 20) and 70\% (14 out of 20), respectively, at 14 days post-challenge. The survival rates of the $p N M B 0315$ and $p N M B 0315+\mathrm{CpG}$ groups were significantly higher compared with the PBS, $p c D N A 3.1(+)$ and CpG control groups $(\mathrm{P}<0.01$; Fig. 5); no significant difference was identified in survival rate was identified between the 

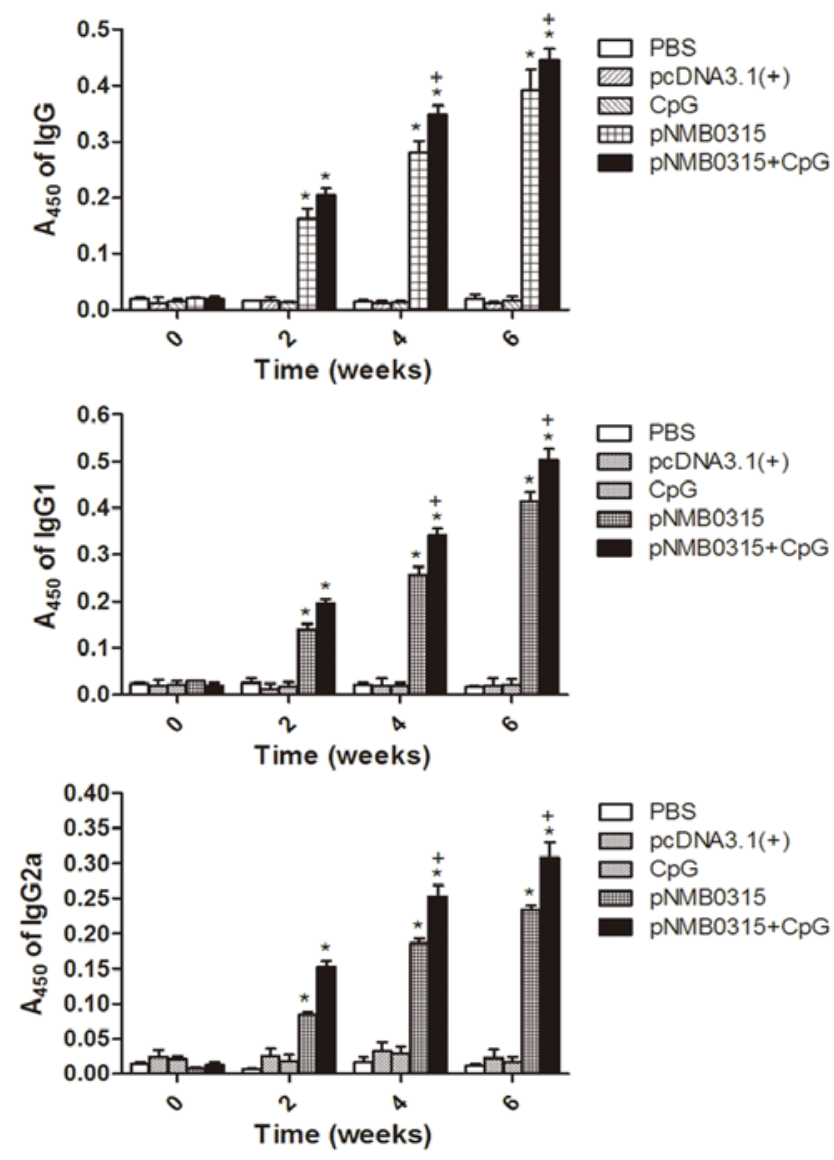

Figure 3. Humoral immune responses induced by $p N M B 0315$. Serum was obtained from the immunized mice as weeks $0,2,4$ and 6 following immunization, and the levels of recombinant NMB0315-specific IgG, IgG1 and $\mathrm{IgG} 2 \mathrm{a}$ antibodies in the immune serum were determined by indirect ELISA method. Data are presented as the mean \pm standard deviation from three independent experiments; ${ }^{\mathrm{P}}<0.01$ vs. $\mathrm{PBS}, \mathrm{CpG}$ and $p c D N A 3.1(+)$ groups; ${ }^{+} \mathrm{P}<0.05$ vs. pNMB0315-only group. Ig, immunoglobulin; NMB, Neisseria meningitidis serogroup B.

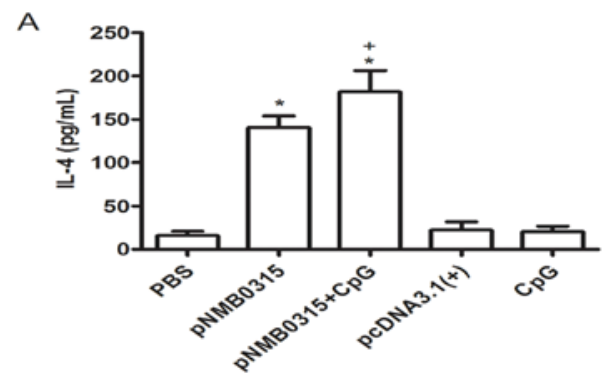

B

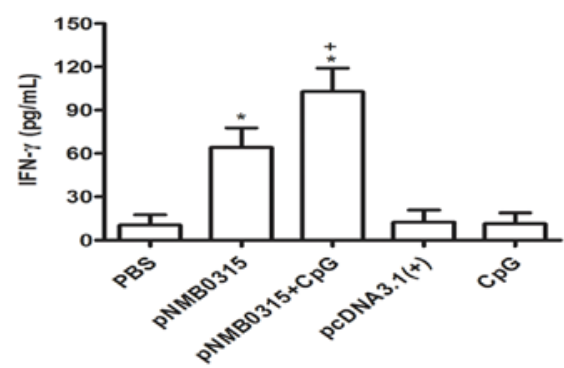

Figure 4. Analysis of cytokine level in pNMB0315-only, $p N M B 0315+\mathrm{CpG}$, pcDNA3.1(+), CpG and PBS groups (A) IL-4 and (B) IFN- $\gamma$ concentrations in the immunized splenic lymphocyte culture supernatants. ${ }^{*} \mathrm{P}<0.01$ vs. $\mathrm{PBS}$, $\mathrm{CpG}$ and $p c D N A 3.1(+)$ groups); ${ }^{+} \mathrm{P}<0.05$ vs. $p N M B 0315$-only group. IFN, interferon; IL, interleukin; NMB, Neisseria meningitidis serogroup B.

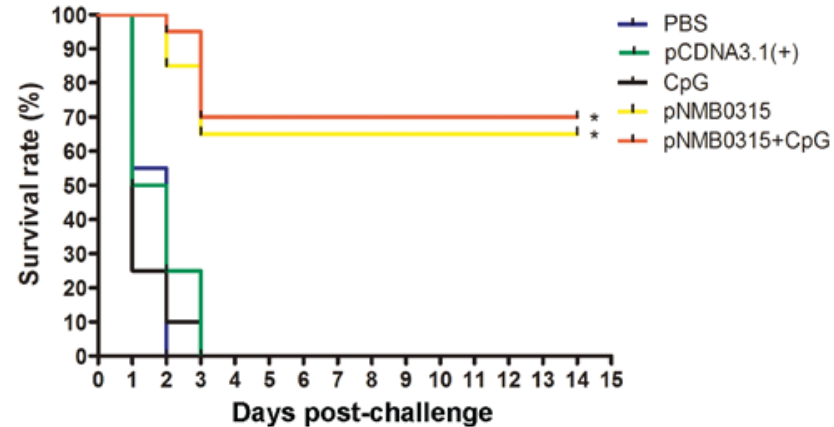

Figure 5. Immunoprotection efficacy against challenge with NMB MC58. The $p N M B 0315$-only and the $p N M B 0315+\mathrm{CpG}$ provided effective immunoprotection with a survival rate of 65 and $70 \%$, respectively, following a lethal dose challenge at 14 days, whereas all mice in the PBS, CpG and $p c D N A 3.1(+)$ control groups succumbed to infection within 3 days of challenge. ${ }^{*} \mathrm{P}<0.01 \mathrm{vs.}$ $\mathrm{PBS}, \mathrm{CpG}$ and $p c D N A 3.1(+)$ groups. NMB, Neisseria meningitidis serogroup B.

pNMB0315 and the $p N M B 0315+\mathrm{CpG}(\mathrm{P}>0.05))$. In addition, SBA demonstrated that the serum bactericidal titers of the $p N M B 0315$ group and the $p N M B 0315+\mathrm{CpG}$ group reached 1:64 and 1:128, respectively (SBA titers $>8$ or a 4 fold increase are considered as protective in many meningococcal species, including group B) (13), following the three immunizations; however, the titers of the control groups were all $<1: 2$ (results not shown). These results indicated that $p N M B 0315$-induced immune serum may have a potent complement-dependent bactericidal activity in vitro and may be highly protective in mice against NMB strain MC58 infection in vivo.

\section{Discussion}

N. meningitides, also known as meningococcus, is a human-specific pathogenic organism that is the cause of encephalomyelitis epidemics $(14,15)$. The most common presentation of invasive meningococcal infection (or meningococcal disease) is meningitis, which results from the spread of the bacterium through the bloodstream. In $\sim 50 \%$ of cases, $N$. meningitides crosses the blood-brain barrier and enters the cerebrospinal fluid, causing purulent meningitis. The mortality rate of meningococcemia is up to $40 \%$ as meningococcemia is more severe than general meningococcal diseases. $(2,6)$. CPS-based vaccines are available and are used for the prevention of infection by $N$. meningitides serogroups A, C, W135 and $\mathrm{Y}$ infections, and the development of an effective vaccine against NMB is important for the prevention and control of the disease (8).

DNA vaccines are a relatively new vaccination strategy. Compared with the traditional vaccines, such as live attenuated or inactivated viral vaccines, or protein subunit vaccines, DNA vaccines offer a number of advantages (16). For example, DNA vaccines are able to replicate and express the protein of interest in the host cells, which is similar to a live attenuated vaccine, but is inherently safer than live attenuated or inactivated viral vaccines. In addition, the expressed protein from DNA vaccines maintains its natural conformation, which potentially produces increased native immunogenicity. Furthermore, DNA plasmids are simple and inexpensive to design and create, and the plasmids themselves are not 
immunogenic (17). The factors associated with the immune effects of a DNA vaccine mainly include regulatory elements, immunization routes and adjuvants. The promoter region is an important regulatory element that directly influences the expression levels of an exogenous gene in vivo. The plasmid used in this study, $p c D N A 3.1(+)$, is a eukaryotic expression vector with a strong promoter from cytomegalovirus, which promotes high-efficiency expression of an exogenous gene in mammalian cells (18). In the present study, a recombinant pNMB0315 was constructed and transfected into eukaryotic cell lines, which effectively expressed rNMB0315 protein, indicating the successful construction of a DNA vaccine. $\mathrm{CpG}$ was used as an adjuvant to enhance immune effects of the DNA vaccine (19). CpGs are recognized by toll-like receptor 9 (TLR9), a receptor found on antigen presenting cells (APCs), which results in the activation of TLR9 and the enhancement of antigen presenting capacity of APCs $(20,21)$.

As $N$. meningitides is an extracellular pathogen, anti-N. meningitides infections are mainly dependent on humoral immunity (22). The DNA vaccine $p N M B 0315$ used in the present study induced high-level NMB0315-specific antibodies $\mathrm{IgG}, \mathrm{IgG} 1$ and $\mathrm{IgG} 2 \mathrm{a}$ in female BALB/c mice. The results indicated that $p N M B 0315$ provided exceptional immunogenicity and that the $\mathrm{CpG}$ adjuvant aided $p N M B 0315$ in eliciting the production of antibodies in mice. It has been reported previously that the subclasses of $\operatorname{IgG}$ present in the serum may reflect the type of immune response, and that $\mathrm{IgG} 2 \mathrm{a}$ was predominant in cellular immunity and IgG1 was predominant in humoral immunity (23). The Th1-type cytokine IFN- $\gamma$ positively correlates with cell-mediated immune responses, which promote the production of $\operatorname{IgG} 2 \mathrm{a}$, whereas the Th2-type cytokine IL-4 is correlated with humoral immune response and promotes the production of IgG1 (24). Serum IgG2a/IgG1 ratios in the $p N M B 0315+\mathrm{CpG}$ and the $p N M B 0315$ groups were $<1$ at weeks 2, 4 and 6 post-inoculation, which suggested that the $p N M B 0315$ DNA vaccine predominantly induced humoral immunity responses.

SBA is used to quantify the levels of antibodies that are specific for bacterial surface determinants and results in complement-mediated lysis of bacteria (25). SBA in vitro is considered as the gold standard test for the evaluation of functional anti-meningococcal antibodies and as an accepted surrogate for protection (26). The serum bactericidal antibody titer of the $p N M B 0315+\mathrm{CpG}$ and the $p N M B 0315$ groups in vitro reached 1:128 and 1:64, respectively, following three immunizations, which may be correlated with the high levels of IgG antibodies in the immunized serum. In addition, the pNMB0315 vaccine exhibited an high immunoprotective efficacy. The vaccine $p N M B 0315+\mathrm{CpG}$ group and $p N M B 0315$ group offered 70 and $65 \%$ protection against NMB MC58, respectively, two weeks following innoculation. The survival rate $(70 \%)$ of $p N M B 0315$ immunization is lower than that of rNspA immunization ( $85 \%$ survival rate) reported by the authors, previously (3), which might be associated with lower transfection efficiency of eukaryotic plasmid $p N M B 0315$. Therefore, a prokaryotic expression vector will be constructed to express recombinant protein NMB0315 and research its immunocompetence and immunoprotection.

In conclusion, the present study successfully constructed and effectively transfected eukaryotic cells with a
pNMB0315 DNA vaccine. pNMB0315 induced high levels of NMB0315-specific IgG, IgG1 and IgG2a antibodies and offered effective immunoprotection against NMB in inoculated mice. In addition, the immune serum containing NMB0315-specific antibodies exhibited strong bactericidal activity, which provided a preliminary proof that the outer membrane protein NMB0315 may be a potential vaccine candidate antigen, and that the $p N M B 0315$ may serve as a promising DNA vaccine against NMB. However, there are a variety of surface-exposed proteins on the MNB that may be associated with bacterial virulence and complicated pathogenesis. It is improbable that the selection of a single virulence factor as a protective antigen may provide complete protection. Therefore, future studies should focus on the development of a multicomponent or multivalent vaccine against NMB.

\section{Acknowledgements}

The present study was supported by The National Natural Science Foundation of China (grant no. 81172890), The Hunan Province Cooperative Innovation Center for Molecular Target New Drug Study (grant no. 2015-351) and The Construct Program of the Key Discipline in Hunan Province and Hunan Provincial Key Laboratory for Special Pathogens Prevention and Control (grant no. 2014-5-2012-312). The authors thank Mrs. Chunxue Lu and Mr. Yukuai Zhang (University of South China, Hengyang, China) for their excellent technical assistance and advice.

\section{References}

1. Shahbaaz M, Bisetty K, Ahmad F and Hassan MI: Towards new drug targets? function prediction of putative proteins of Neisseria meningitidis MC58 and their virulence characterization. OMICS 19: 416-434, 2015.

2. Silva GP, Cruz SC, Cruz AC and Milagres LG: Short-term and long-term antibody response by mice after immunization against Neisseria meningitidis B or diphtheria toxoid. Braz J Med Biol Res 46: 148-153, 2013

3. Ying S, He J, Yu M, Zhang Y, Deng S, Zhang L, Xie M and Hu S: Recombinant Neisseria surface protein A is a potential vaccine candidate against Neisseria meningitides serogroup B. Mol Med Rep 10: 1619-1625, 2014.

4. Pelton SI: The global evolution of meningococcal epidemiology following the introduction of meningococcal vaccines. J Adolesc Health 59 (Suppl 2): S3-S11, 2016.

5. Braunstein M, Rajkumar P, Claus CL, Vaccarelli G, Moore AJ, Wang D and Anderson MK: HEBAlt enhances the T-cell potential of fetal myeloid-biased precursors. Int Immunol 22: 963-972, 2010.

6. Brehony C, Rodrigues CM, Borrow R, Smith A, Cunney R, Moxon ER and Maiden MCJ: Distribution of bexsero ${ }^{\circledR}$ antigen sequence types (BASTs) in invasive meningococcal disease isolates: Implications for immunisation. Vaccine 34: 4690-4697, 2016.

7. Sung JW, Hsieh SY, Lin CL, Leng CH, Liu SJ, Chou AH, Lai LW, Lin LH, Kwok Y, Yang CY and Chong P: Biochemical characterizations of Escherichia coli-expressed protective antigen Ag473 of Neisseria meningitides group B. Vaccine 28: 8175-8182, 2010.

8. Khatami A and Pollard AJ: The epidemiology of meningococcal disease and the impact of vaccines. Expert Rev Vaccines 9: 285-298, 2010.

9. Martin D, Brodeur BR, Hamel J, Couture F, de Alwis U, Lian Z, Martin S, Andrews D and Ellis RW: Candidate Neisseria meningitidis NspA vaccine. J Biotechnol 83: 27-31, 2000.

10. Wang X, Yang X, Yang C, Wu Z, Xu H and Shen Y: Crystal structure of outer membrane protein NMB0315 from Neisseria meningitidis. PLoS One 6: e26845, 2011. 
11. Lee J, Kang HE and Woo HJ: Protective immunity conferred by the C-terminal fragment of recombinant Pasteurella multocida toxin. Clin Vaccine Immunol 19: 1526-1531, 2012.

12. Fang Y, Lin H, Ma Z and Fan H: Construction and immunogenicity of recombinant swinepox virus expressing outer membrane protein L of salmonella. J Microbiol Biotechnol 26: 1173-1181, 2016.

13. Borrow R, Carlone GM, Rosenstein N, Blake M, Feavers I, Martin D, Zollinger W, Robbins J, Aaberge I, Granoff DM, et al: Neisseria meningitidis group B correlates of protection and assay standardization-International Meeting Report Emory University, Atlanta, Georgia, United States, 16-17 March 2005. Vaccine 24: 5093-5107, 2006.

14. Johansson L, Rytkonen A, Bergman P, Albiger B, Källström H, Hökfelt T, Agerberth B, Cattaneo R and Jonsson AB: CD46 in meningococcal disease. Science 301: 373-375, 2003.

15. Sjölinder $\mathrm{H}$ and Jonsson AB: Olfactory nerve-a novel invasion route of Neisseria meningitidis to reach the meninges. PLoS One 5: e14034, 2010.

16. Chaudhari A, Pathakota GB and Annam PK: Design and construction of shrimp antiviral DNA vaccines expressing long and short hairpins for protection by RNA interference. Methods Mol Biol 1404: 225-240, 2016.

17. Abdulhaqq SA and Weiner DB: DNA vaccines: Developing new strategies to enhance immune responses. Immunol Res 42: 219-232, 2008.

18. Wu QQ, Zhang QH and Ma L: Construction of recombinant eukaryotic expression plasmid pcDNA3.1(+)-mtDNA of human colorectal carcinoma cells. Di Yi Jun Yi Da Xue Xue Bao 25: 1016-1019, 2005 (In Chinese).

19. Cheng WK, Plumb AW, Lai JC, Abraham N and Dutz JP: Topical $\mathrm{CpG}$ oligodeoxynucleotide adjuvant enhances the adaptive immune response against influenza A infections. Front Immunol 7: 284, 2016.
20. Hemmi H, Takeuchi O, Kawai T, Kaisho T, Sato S, Sanjo H, Matsumoto M, Hoshino K, Wagner H, Takeda K and Akira S: A Toll-like receptor recognizes bacterial DNA. Nature 408: 740-745, 2000.

21. Wu HM, Wang J, Zhang B, Fang L, Xu K and Liu RY: CpG-ODN promotes phagocytosis and autophagy through JNK/P38 signal pathway in Staphylococcus aureus-stimulated macrophage. Life Sci 161: 51-59, 2016.

22. Buchanan RM, Briles DE, Arulanandam BP, Westerink MA, Raeder RH and Metzger DW: IL-12-mediated increases in protection elicited by pneumococcal and meningococcal conjugate vaccines. Vaccine 19: 2020-2028, 2001.

23. Gong W, Qi Y, Xiong X, Jiao J, Duan C and Wen B: Rickettsia rickettsii outer membrane protein $\mathrm{YbgF}$ induces protective immunity in $\mathrm{C} 3 \mathrm{H} / \mathrm{HeN}$ mice. Hum Vaccin Immunother 11: 642-649, 2015.

24. Trotter CL, Yaro S, Njanpop-Lafourcade BM, Drabo A, Kroman SS, Idohou RS, Sanou O, Bowen L, Findlow H, Diagbouga S, et al: Seroprevalence of bactericidal, specific $\mathrm{IgG}$ antibodies and incidence of meningitis due to group A Neisseria meningitidis by age in Burkina Faso 2008. PLoS One 8: e55486, 2013.

25. Bash MC, Lynn F, Mocca B, Borrow R, Findlow H, Hassan-King M, Preziosi MP, Idoko O, Sow S, Kulkarni P and Laforce FM: Development and use of a serum bactericidal assay using pooled human complement to assess responses to a meningococcal group A conjugate vaccine in African toddlers. Clin Vaccine Immunol 21: 755-761, 2014

26. Findlow J, Holland A, Andrews N, Weynants V, Sotolongo F, Balmer P, Poolman J and Borrow R: Comparison of phenotypically indistinguishable but geographically distinct Neisseria meningitidis group B isolates in a serum bactericidal antibody assay. Clin Vaccine Immunol 14: 1451-1477, 2007. 